\title{
An updated explanation of ancestral karyotype changes and reconstruction of evolutionary trajectories to form Camelina sativa chromosomes
}

Zhikang Zhang ${ }^{1 \dagger}$, Fanbo Meng ${ }^{1 \dagger}$, Pengchuan Sun ${ }^{1}$, Jiaqing Yuan², Ke Gong ${ }^{1}$, Chao Liu', Weijie Wang ${ }^{1 *}$ and Xiyin Wang ${ }^{1,3^{*}}$ (D)

\begin{abstract}
Background: Belonging to lineage I of Brassicaceae, Camelina sativa is formed by two hybridizations of three species (three sub-genomes). The three sub-genomes were diverged from a common ancestor, likely derived from lineage I (Ancestral Crucifer karyotype, ACK). The karyotype evolutionary trajectories of the C. sativa chromosomes are currently unknown. Here, we managed to adopt a telomere-centric theory proposed previously to explain the karyotype evolution in C. sativa.

Results: By characterizing the homology between A. lyrata and C. sativa chromosomes, we inferred ancestral diploid karyotype of $C$. sativa (ADK), including 7 ancestral chromosomes, and reconstructed the evolutionary trajectories leading to the formation of extant $C$. sativa genome. The process involved 2 chromosome fusions. We found that sub-genomes Cs-G1 and Cs-G2 may share a closer common ancestor than Cs-G3. Together with other lines of evidence from Arabidopsis, we propose that the Brassicaceae plants, even the eudicots, follow a chromosome fusion mechanism favoring end-end joining of different chromosomes, rather than a mechanism favoring the formation circular chromosomes and nested chromosome fusion preferred by the monocots.
\end{abstract}

Conclusions: The present work will contribute to understanding the formation of C. sativa chromosomes, providing insight into Brassicaceae karyotype evolution.

Keywords: Brassicaceae, C. sativa, Chromosome, Karyotype, Polyploid

\section{Background}

Brassicaceae (mustard family) is one of the largest groups in plants, being composed of an approximate 3709 species, classified into 338 genera [1]. It includes several species of prominent scientific and economic importance. According to phylogenetic relationship, Camelineae species (Arabidopsis thaliana, Arabidopsis lyrata,

\footnotetext{
*Correspondence: weijiewang@yahoo.com; wang.xiyin@gmail.com

'Zhikang Zhang and Fanbo Meng contributed equally to this work.

'School of Life Sciences, North China University of Science and Technology, Tangshan 063210, Hebei, China

Full list of author information is available at the end of the article
}

and Capsella rubella) and Brassica species (Brassica rapa, Brassica nigra and Brassica oleracea) respectively represent lineage I and lineage II, two of three wellsupported lineages among the Brassicaceae $[2,3]$.

With the rapid increase of Brassicaceae genome assemblies, reconstructing ancestral genome can help understand the evolutionary history of the extant Brassicaceae families and species. With genetic maps of $A$. lyrata and C. rubella, Schranz et al. defined 24 conversed GBs (labelled as A-X) related to ancestral karyotype $(\mathrm{AK}, n=8)[4]$. Ancestral crucifer karyotype (ACK, $\mathrm{n}=$

C C The Author(s). 2020 Open Access This article is licensed under a Creative Commons Attribution 4.0 International License, which permits use, sharing, adaptation, distribution and reproduction in any medium or format, as long as you give appropriate credit to the original author(s) and the source, provide a link to the Creative Commons licence, and indicate if changes were made. The images or other third party material in this article are included in the article's Creative Commons licence, unless indicated otherwise in a credit line to the material. If material is not included in the article's Creative Commons licence and your intended use is not permitted by statutory regulation or exceeds the permitted use, you will need to obtain permission directly from the copyright holder. To view a copy of this licence, visit http://creativecommons.org/licenses/by/4.0/. The Creative Commons Public Domain Dedication waiver (http://creativecommons.org/publicdomain/zero/1.0/) applies to the data made available in this article, unless otherwise stated in a credit line to the data. 
8), improved from $\mathrm{AK}$, is recognized as ancestral state of lineage I (Fig. 1 and 4a), based on the fact that most base common number of chromosomes is eight [5]. Besides, they reconstructed Proto-Calepineae karyotype (PCK, $n=7)$ as ancestral karyotype of 6 Brassicaceae tribes (Fig. 1). While PCK is inherited in three of the six tribes (Calepineae, Conringieae, and Noccaeeae), which belongs to lineage II, the rest three tribes (Eutremeae, Isatideae, and Sisymbrieae) is characterized by an additional translocation comparing to PCK, which is referred as translocation Proto-Calepineae Karyotype (tPCK, $n=7$ ). Cheng et al. provided evidence that tPCK represents ancestral karyotype of the mesohexaploid $B$. rapa, the genus Brassica, and the tribe Brassiceae, by comparing three ancestral sub-genomes of Chinese cabbage $(B$. rapa) with PCK and tPCK [6].

Running through the evolutionary history of plant kingdom, polyploidization continually led to genome doubling/tripling, genome repatterning, and gene loss, characterizing genome instability and fractionation [79]. Interestingly, chromosome numbers could be much reduced to a kind of normal range after rounds of polyploidization. After two extra Brassicaceae-common duplications (BCD) [10], A. thaliana has only five base chromosomes. It was proposed that chromosome number reduction (CNR) was often the result of reciprocal translocations, which combined two chromosomes into a larger one and a smaller one, and the smaller chromosome got lost during meiosis [11]. For example, ACK and PCK shared the same karyotype of five chromosomes (AK1-4, and 7), AK6/8 and AK5/6/8 in PCK formed by reciprocal translocations between AK5, AK6 and $\mathrm{AK} 8$, resulting in chromosome number reduction from eight to seven [5]. Notably, an alternative telomere-centric model suggests that the removal of telomeres caused chromosome fusion and chromosome number reduction during the karyotype evolution, and explained the molecular dynamics of chromosome reformation [12]. Based on the telomere-centric model, ancestral karyotypes and evolutionary trajectories of chromosomes were reconstructed for Arabidopsis, grasses, and legumes [12-14].

Belonging to lineage I of Brassicaceae [3] (Fig. 1), C. sativa (false flax) is a high-quality oilseed crop with several advantages of high production and resistance to drought and diseases for industrial production of biodiesel [15]. It was proposed that $C$. sativa represented a whole-genome triplication event relative to $A$. thaliana [16], and three sub-genomes were defined (Cs-G1, CsG2 and Cs-G3) [17] (Fig. 2). The sub-genomes CsG1and Cs-G2 are more closely related to each other than any of the diploids assayed based on phylogenetic relationship, Cs-G3 shows a clear expression level advantage over the other two sub-genomes, and the three sub-genomes have an almost identical Ks distribution of synteny genes with $A$. thaliana. The three sub-genomes were likely diverged from a common ancestor and the extant $C$. sativa hexaploidy genome result from a twostage allopolyploid pathway [17]. The genus Camelina contains approximate 6 species, including $C$. sativa $(2 \mathrm{n}=6 \mathrm{x}=40)$, Camelina microcarpa $(2 \mathrm{n}=12,2 \mathrm{n}=4 \mathrm{x}=$ 26, $2 \mathrm{n}=6 \mathrm{x}=40)[18]$, Camelina hispida $(2 \mathrm{n}=2 \mathrm{x}=14)$, Camelina rumelica $(2 \mathrm{n}=4 \mathrm{x}=26)$, Camelina neglecta $(2 \mathrm{n}=2 \mathrm{x}=12)$ [19], Camelina laxa [20] $(2 \mathrm{n}=2 \mathrm{x}=12)$. By phylogenetic analyses of a set of unanchored genome scaffolds, it was proposed that one C. microcarpa accession $(2 \mathrm{n}=26)$ included the two sub-genomes of $C$. sativa (Cs-G1 and Cs-G2), showing that the C. microcarpa

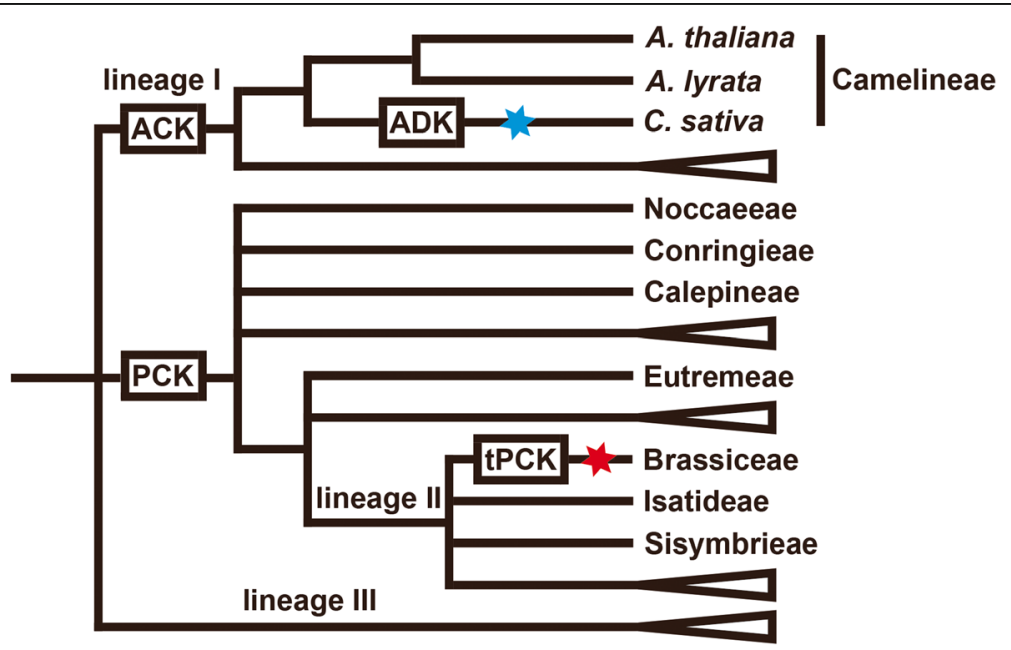

Fig. 1 Phylogenetic relationship of selected tribes and species within the Brassicaceae. The red star refers to the Brassiceae-specific wholegenome triplication and blue star refers to a whole-genome triplication event relative to the crucifer model $A$. thaliana. Adopted and modified from Mandáková and Lysak (2008), Franzke et al. (2011) and Cheng et al. (2013) 


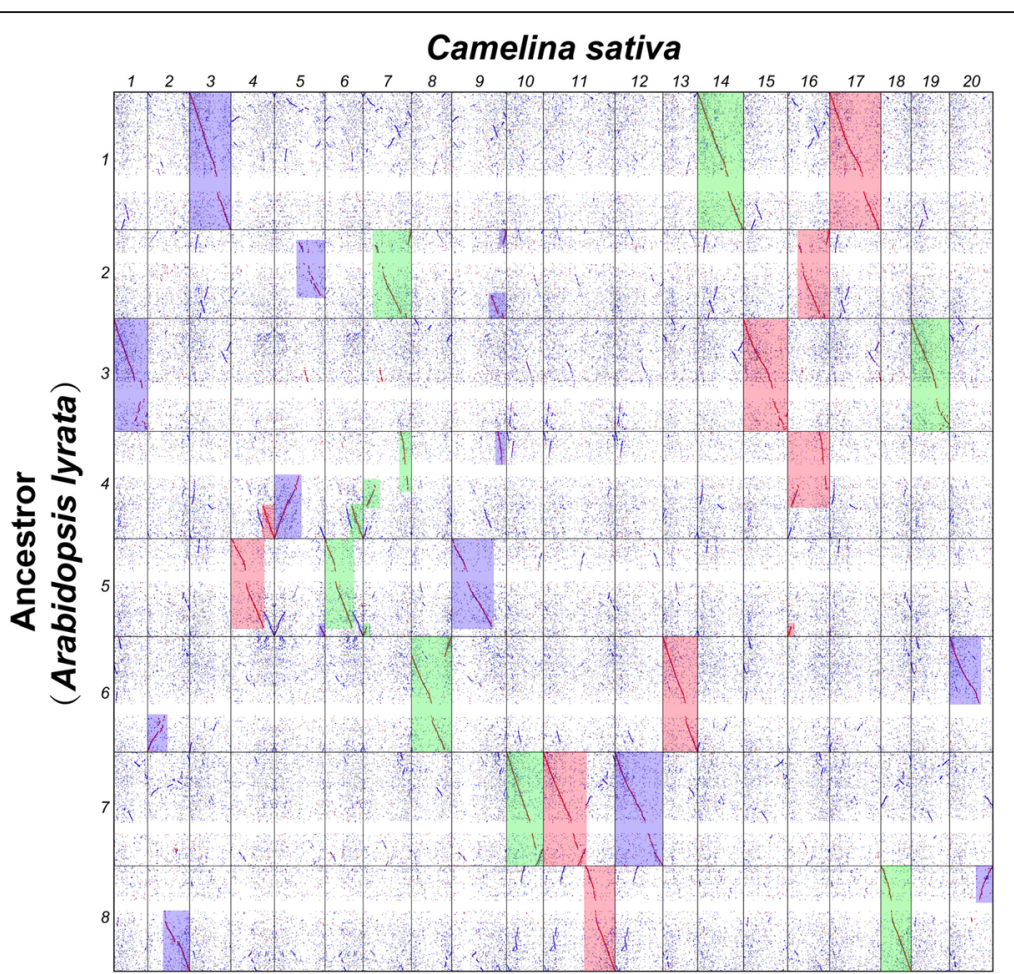

Fig. 2 Dot-plot of homologous genes comparing A. lyrata and C. sativa genomes. C. sativa genome is divided into three sub-genomes which are filled in with red (Cs-G1), green (Cs-G2) and blue (Cs-G3) according to syntenic regions. Adopted and modified from Kagale at al. (2014)

may be the crop's wild ancestor. The third sub-genome shares significant homology to C. hispida $(2 \mathrm{n}=14)$, implying this may represent an extant progenitor of the sub-genome (Cs-G3) [21].

The ancestral diploid karyotype of C. sativa (derivative of ACK, dACK) were reconstructed based on synteny and colinearity between C. sativa and Arabidopsis species [17]. However, dynamic changes of their formation or evolutionary trajectories of extant $C$. sativa chromosomes have not been well inferred. Here, using the theory of telomere-centric genome repatterning, we inferred a different ancestral diploid karyotype of $C$. sativa (ADK), and compared to previous inference, and reconstructed the ancestral karyotypes and evolutionary trajectories of the extant C. sativa genome. The present work will contribute to understanding the formation and evolution of the chromosomes in C. sativa and other Brassicaceae plants.

\section{Results}

Inference of ancestral diploid karyotype of $C$. sativa

To understand the evolutionary trajectories of ADK before divergence of three $C$. sativa sub-genomes, we analyzed the syntenic conservation and chromosome repatterning between the genomes of the ancestor of lineage I and $C$. sativa. Here, we took the A. lyrata genome as the reference of ancestral genome of lineage I for the sake of the significant colinearity between their genomes (Fig. S1 and Table S1) and high similarity between their karyotype. By searching homologous genes between them, we drew homologous gene dot-plots (Figs. 2 and 3), and showed orthologous correspondence between ancestral genomes of lineage I and C. sativa genomes.

In the homologous gene dot-plots of the two genomes, produced directly by using BLASTP hits and further highlighted by integrating inferred colinear genes, every chromosome in the ancestral genome has three homoeologous chromosomes or groups of homoeologous chromosome regions in $C$. sativa genome. We found that 5 ACK chromosomes had nearly perfect orthologous correspondence with at least one or more complete chromosomes in C. sativa (Fig. 3a, b, c, d, and e), showing that the integrity of each of these 5 chromosomes in ADK (correspondingly defined as ADK chromosomes 1 , $2,5,6,7)$, which directly inherited the chromosome structure of ACK (AK chromosomes 1, 3, 6, 7, 8) without prominent DNA rearrangements.

Notably, orthologous correspondence between AK2, 4, 5 and Cs4, 16 (Cs-G1) is nearly the same as that between AK2, 4, 5 and Cs6, 7 (Cs-G2) (Fig. 3g and h), indicating that Cs-G1 and Cs-G2 shared two ancestral chromosomes, which majorly formed through reciprocal translocation of arms (RTA) and end-end joining (EEJ) between AK2, 4, 5. By searching shared gene synteny 

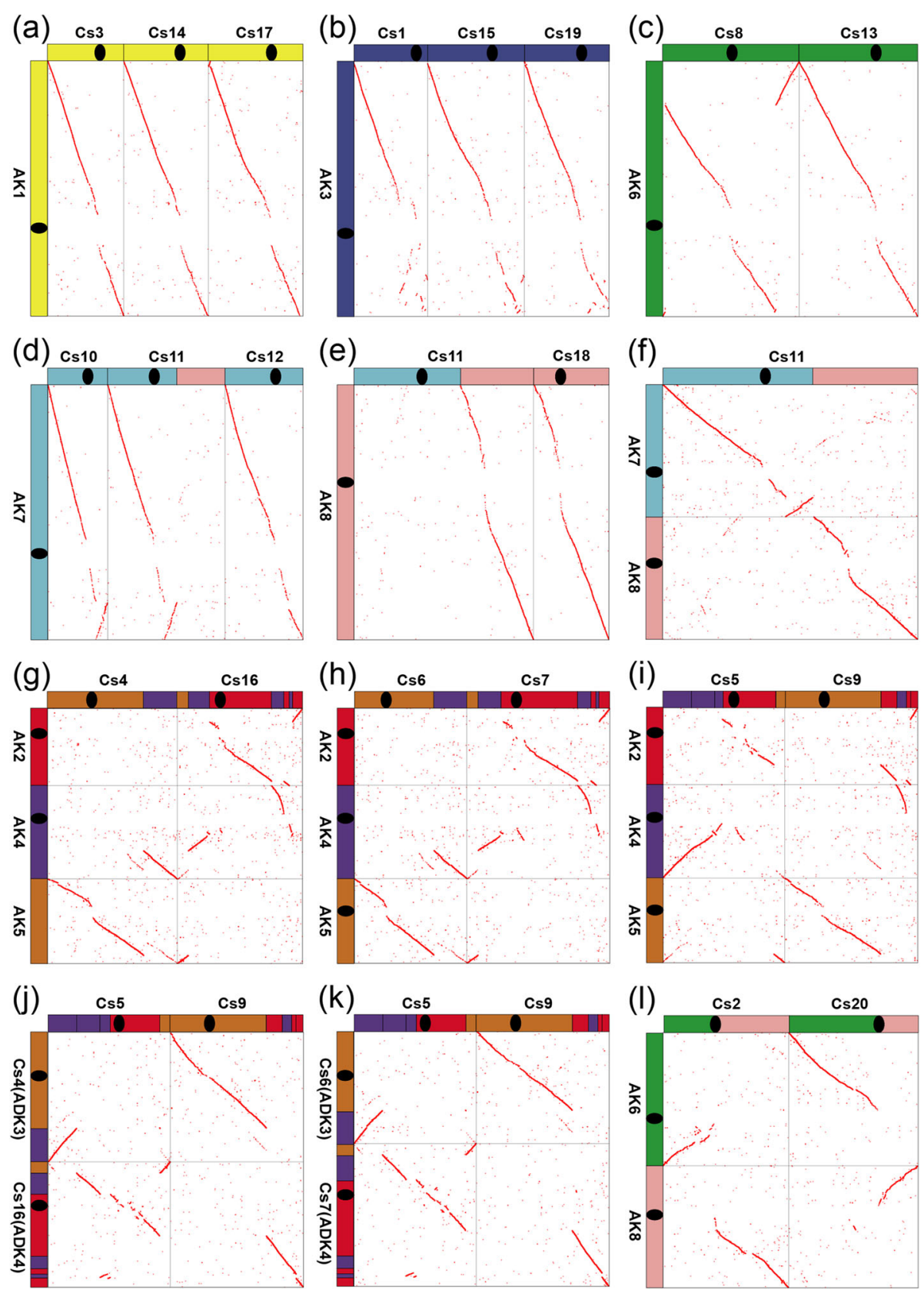

Fig. 3 Homologous dot-plots between selected A. lyrata or C. sativa and C. sativa chromosomes. Cs, C. sativa. a formation of Cs3, 14, 17; b formation of Cs1, 15, 19; $\mathbf{c}$ formation of Cs8, 13; $\mathbf{d}$ formation of Cs10, 12; e formation of Cs18; $\mathbf{f}$ formation of Cs11; $\mathbf{g}$ formation of Cs4, 16; $\mathbf{h}$ formation of Cs6, 7; $\mathbf{i}, \mathbf{j}$ and $\mathbf{k}$ formation of Cs5, 9; I formation of Cs2, 20

between $A$. lyrata and $C$. sativa genomes, we further found that the crossing-over positions between chromosomes $(\mathrm{AK} 4,5)$ were respectively between gene AL482377 (Corresponding C. sativa ortholog: Csa16g006880.1) and AL321151 (Csa04g046610.1) in AK4, and that between gene AL486375 (Csa04g046590.1) and AL486377 (Csa16g006870.1) in AK5. Actually, the following two evolutionary trajectories could explain the changes of these chromosomes. A relatively more complex evolutionary trajectory could occur as follows: AK2 and AK4 crossed over near one telomere of each of them, resulting in EEJ to produce AK2/4 and formation of a satellite chromosome of two telomeres (and possibly little DNA); then cross-over between AK5 and neo-AK2/4, which experienced one extra translocation and pericentric inversion, resulting in RTA between the two chromosomes to produce AK5/4 (ADK3) and ADK2/4/5 (ADK4) (Fig. 4c). An alternative trajectory could occur as follows: a cross-over 
(a)

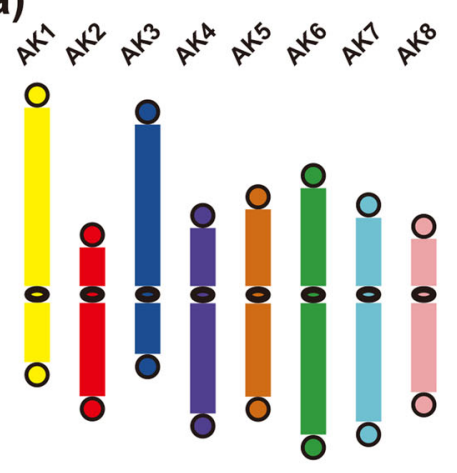

(b)

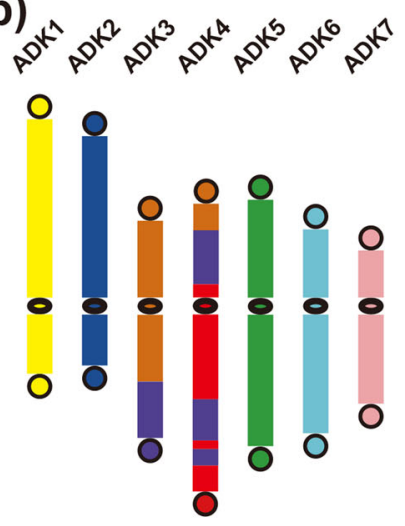

(c)

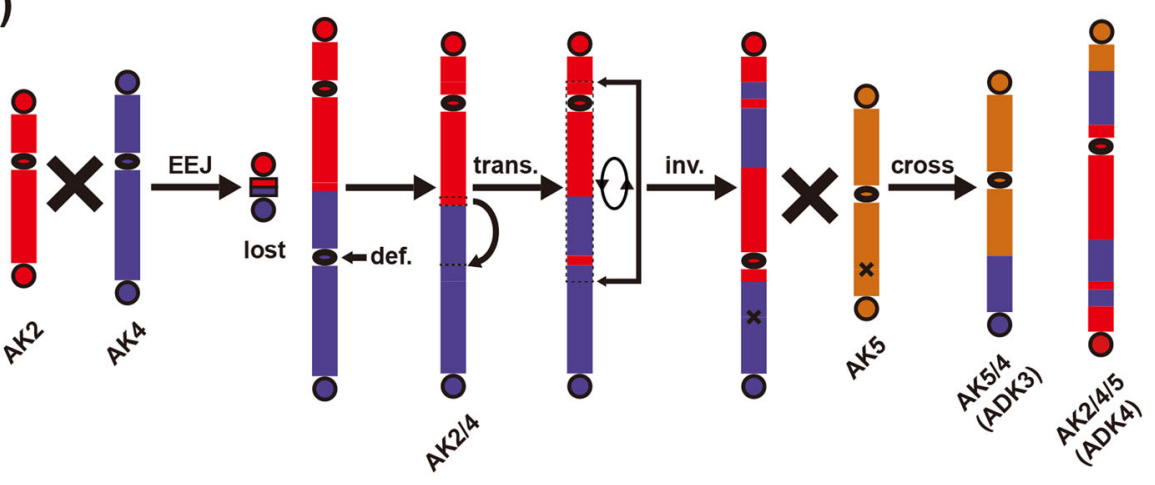

(d)

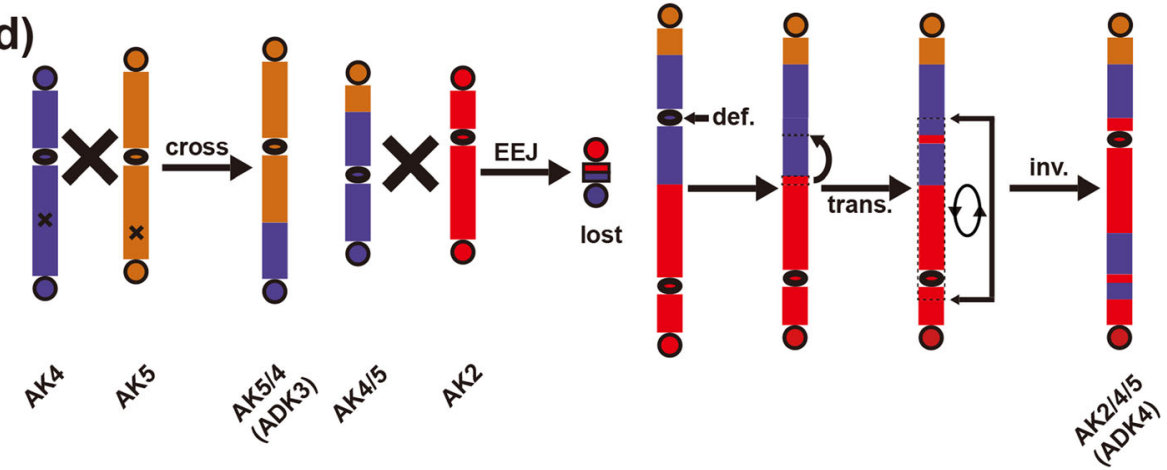

Fig. 4 Evolutionary trajectories from ACK to ADK. a Ancestral Crucifer Karyotype (ACK). b Ancestral Diploid Karyotype of C. sativa (ADK). c, d two comparative evolutionary trajectories of chromosomes ADK3, 4

between AK4 and AK5 resulted in reciprocal translocation of arms (RTA) to produce AK5/4, forming ADK3, and intermediate AK4/5. Then, AK4/5 and AK2 crossed over near one telomere of each of them, resulting in chromosome end-end joining (EEJ) to produce AK2/4/5 and likely formation of a satellite chromosome by two telomeres (and possibly little DNA). The neo-chromosome AK2/4/5 experienced one extra translocation and pericentric inversion to form ADK4 (Fig. 4d). No matter which trajectory was the actual one, the satellite chromosome likely produced was lost, eventually reducing the chromosome number from 8 in ACK to 7 in ADK.
Orthologous correspondence between AK2, 4, 5 and Cs5, 9 (Cs-G3) (Fig. 3i) is much different from that between AK2, 4, 5 and Cs4, 16 (Cs-G1) or Cs6, 7 (Cs-G2), showing that Cs5, 9 has particular structures not shared with the other two sets of chromosomes (Cs4, 16 and Cs6, 7). It seems that Cs-G3 does not share the two ancestral chromosomes (ADK3, 4) with Cs-G1 and Cs-G2. However, orthologous correspondence between Cs4, 16 or Cs6, 7 and Cs5, 9 (Fig. 3j and k), showing that Cs5, 9 are majorly formed by RTA between ADK3 and ADK 4 (Fig. 5). By searching gene synteny between $A$. lyrata and $C$. sativa genomes, we further characterized the 


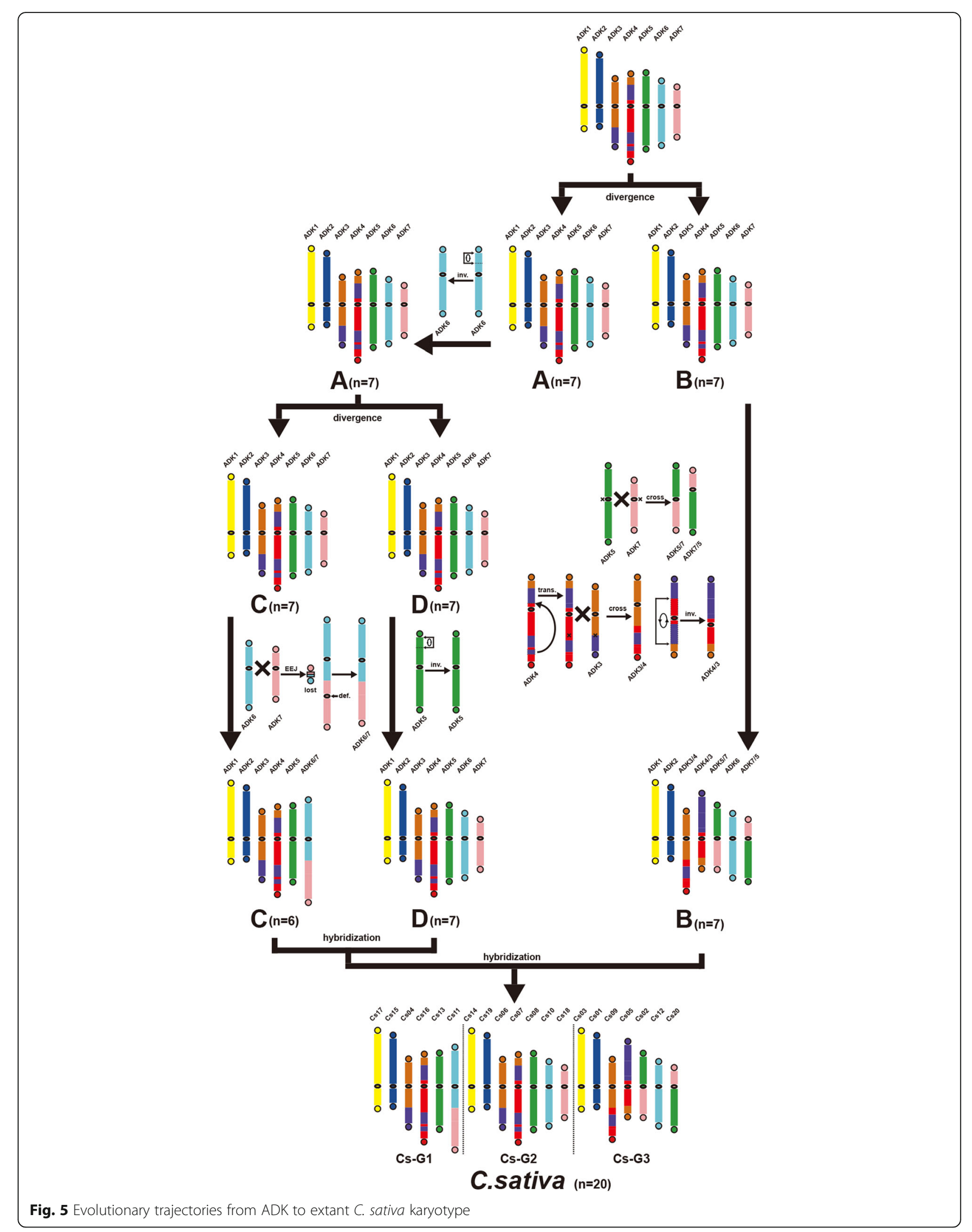


crossing-over positions between chromosomes (ADK3, 4) are respectively between gene AL486375 (Corresponding C. sativa ortholog: Csa04g046590.1) and AL321151 (Csa04g046610.1) in ADK3 (where chromosome arms of AK4, 5 combined), and that between gene AL476152 (Csa09g071500.1) and AL926342 (Csa09g071510.1) in ADK4. This findings provide a clear evidence to support that the Cs-G3 actually inherited karyotype structures of the two ancestral chromosomes (ADK3, 4), which are shared with Cs-G1 and Cs-G2.

\section{Inferring evolutionary trajectories from ADK to extant $C$. sativa karyotype}

Shared chromosome structural patterns can help understand phylogenomic relationship. In homologous gene dot-plots, orthologous correspondence between AK7 (ADK6) and Cs10, 11, 12 (Fig. 3d) suggested that one paracentric inversion is common to Cs10 and Cs11, respectively corresponding to Cs-G1 and Cs-G2, respectively, but not in chromosome Cs12 from Cs-G3. It suggested that Cs-G1 and Cs-G2 are not directly diverged from $\mathrm{ADK}$, but share a common ancestor with one paracentric inversion as compared to ADK6.

The formation process of the three sub-genomes and C. sativa genomes could occur as follows: the ancestral diploid of C. sativa differentiated into species A and B firstly, and then species A differentiated into species $C$ and $\mathrm{D}$ after one paracentric inversion occurred in ADK6 (Fig. 5). Crossing-over between ADK6 and ADK7 occurred near one telomere of each chromosome in species $\mathrm{C}$, resulting in chromosome end-end joining (EEJ) to produce ADK6/7 and formation of a satellite chromosome of two telomeres and little DNA. ADK5 in species $\mathrm{D}$ experienced one paracentric inversion independently (Fig. 3c and 5). Crossing-over occurred between ADK3 and ADK4 in species B, which experienced one translocation, resulting in reciprocal translocation of arms (RTA) to produce ADK3/4 and ADK4/3, which experienced one pericentric inversion (Fig. $3 \mathbf{j}$ and 5 ). RTA between ADK5 and ADK7 in species B occurred to produce ADK5/7 and ADK7/5 (Fig. 3j and 5). The crossing-over positions between chromosomes (ADK5, 7) are respectively between gene AL489681 (Corresponding C. sativa ortholog: Csa20g058860.1) and AL351869 (Csa02g002270.1) in ADK5 (the region where the centromere of ADK5 is located), and that between gene AL494932 (Csa20g041660.1) and AL494934 (Csa02g033470.1) in ADK7 (the region where the centromere of ADK7 is located). An initial hybridization event between species C (Cs-G1) and D (Cs-G2), resulting in a tetraploid genome, followed by an additional hybridization event between the tetraploid genome and species B (Cs-G3), eventually forming the extant hexaploid genome of C. sativa [17] (Fig. 5).
During the formation of the karyotype of C. sativa, 14 chromosomes of $C$. sativa inherited the chromosome structures of ADK ones. While one paracentric inversion occurred in Cs-G2 to produce one new chromosome, two RTAs occurred in Cs-G3 with one translocation and pericentric inversion to produce four new chromosomes. EEJ occurred in Cs-G1 to produce one new chromosome and one satellite chromosome. The loss of the satellite chromosomes resulted in the chromosome number reduction from 21 to 20 .

\section{Discussion}

A telomere-centric theory shows the likely karyotype changes likely involve the production of free-end chromosomes, which were eventually inserted into other chromosomes, and/or the end-ending joining of different chromosomes $[12,13]$. Actually, chromosome may form a circular form and cross-over may occur near its two telomeres, and the resolution of the cross-over may produce a telomere-free chromosome and a satellite chromosome of two telomeres and little DNA; the telomere-free chromosome may invade another chromosome and eventually result in the merge of the invading one into the invaded one, referred to nested chromosome fusion (NCF). Alternatively, two chromosomes may cross over near one telomere of each chromosome, resulting in chromosome end-end joining (EEJ) and formation of a satellite chromosome. Besides, reciprocal translocation of arms (RTA) may although occur. The loss of satellite chromosome explains chromosome number reduction. Here, we used the telomere-centric model to update the explanation of the Brassicaceae karyotype evolution.

Though karyotypes that we inferred are of the same chromosome numbers in key evolutionary nodes as in previous [17], the karyotypes or the chromosome formations are updated. The ancestral diploid karyotype of $C$. sativa, inferred by previous study, only involved chromosome correspondence between AK2 and AK4, but ignores that AK5 should have also taken part in the formation of the ADK3 and ADK4, which is strongly suggested in homologous gene dot-plots between A. lyrata and C. sativa genomes (Fig. 3j, h; Fig. 4). Comparing to previous study [17], we further inferred the evolutionary trajectories from ADK to extant $C$. sativa karyotype, which involved one EEJ and two RTAs. Previously, the three sub-genomes of $C$. sativa were regarded as having no genome fractionation bias [17]. However, the sharing two ancestral chromosomes by Cs-G1 and Cs-G2, having evolved from ADK chromosomes, provided clear evidences to show their higher similarity and less fractionation as compared to the other chromosome.

Chromosome number reduction (CNR) in Brassicaceae plants took always the end-end-joining or EEJ mechanism rather than the nested-chromosome fusion 
or NCF mechanism. NCF and EEJ, which can generate satellite chromosome(s), the loss of which resulted in the CNR. Interestingly, the occurrence of the two mechanisms of CNR always showed an obvious plant family preference. The number of occurrences of the two mechanisms in grass family is summerized as follows: from the common 12 ancestral chromosomes, 7 NCFs and $0 \mathrm{EEJ}$ occurred to produce 5 extant Brachypodium chromosomes, $5 \mathrm{NCFs}$ and $1 \mathrm{EEJ}$ to form wheat chromosomes, $1 \mathrm{NCF}$ and $0 \mathrm{EEJ}$ to form foxtail millet chromosomes, 13 NCFs and 4 EEJs to form maize chromosomes. In summary, there are $23 \mathrm{NCFs}$ and 5 EEJs occurring independently to form extant grass chromosomes, showing NCFs were significantly more preferred than EEJ (Chisq-test $P$-value $\approx 0.02395$ ). In contrast, the CNR during the formation of $A$. thaliana chromosomes from eight ancestral chromosomes involved only three EEJs but not NCF [12]. Similar to $A$. thaliana, the formation of ADK, and the formation of the extant hexaploid genome of C. sativa, EEJ is the only mechanism that causes CNR. This shows an exclusive preference of EEJ in Brassicaceae. A significant preference of EEJ over NCF was also observed in legumes. Though the sampled families are still too limited, it seems that eudicots prefer EEJ, and monocots prefer NCF, resulting in CNR.

While homologous gene dot-plots are always used to infer gene colinearity in a genome or between genomes, and multiple layers of gene colinearity would suggest the occurrence and ploid levels of polyploidization [10, 22]. Using the assistance of homologous gene dot-plots, it was shown that the cucurbits shared a tetraploid ancestor overlooked by multiple genome sequencing efforts [22], highlighting its unelectable values in genome structure analysis. Besides, homologous gene dot-plots can also intuitively show chromosome changes and trace of genome repatterning [12-14]. A recent effort characterized gene colinearity between more than ten legumes and reconstructed the karyotypes of ancestral nodes during the divergence of legumes and evolutionary trajectories of legume chromosomes [14]. Here, we exploited the gene colinearity patterns in homologous dot-plots and inferred karyotype evolution and even phylogenetic relationship in Brassicaceae plants, further consolidating its usage in ancestral karyotype inference.

\section{Conclusions}

By using the telomere-centric model, we inferred ancestral diploid karyotype of $C$. sativa (ADK), including 7 ancestral chromosomes, and reconstructed the karyotype evolutionary trajectories leading to the formation of $C$. sativa genome. The process involved 2 chromosome fusions. By the analysis of chromosome structure and karyotype evolution, we found that sub-genomes Cs-G1 and Cs-G2 may share a closer common ancestor than Cs-G3. The present work will contribute to understanding the formation and evolution of the chromosomes in C. sativa and other Brassicaceae plants.

\section{Methods \\ Plant genome data sets}

The genomes of $A$. lyrata [23] and C. sativa [17] were downloaded from NCBI (https://ftp.ncbi.nlm.nih.gov/genomes/all/GCF/000/004/255/GCF_000004255.2_v.1.0, https://ftp.ncbi.nlm.nih.gov/genomes/all/GCF/000/633/ 955/GCF_000633955.1_Cs).

\section{Dot-plot generation}

We used BLASTP [24] to search for homologous pairs (E-value $<1 \times 10^{-5}$ ) between every possible pair of chromosomes in two genomes. The best, second best, and other matches with E-value $>1 \mathrm{e}-5$ were displayed in different colors, to help distinguish orthology from paralogy, or layers of paralogy as a result of recursive WGD events. Dot-plots were produced using home-made Python scripts.

\section{Circos diagram generate and inferring positions of breakpoints}

Homologous pairs detected by BLASTP were used as input for ColinearScan 1.01 [25] to obtain syntenic regions between $A$. lyrata and $C$. sativa genomes. The maximum gap length $(\mathrm{mg})$ was set to be 50 intervening genes between neighboring genes in colinearity on both chromosomes. Circos diagram of the two genome were produced by TBtools [26] based on the colinearity. Searching the syntenic regions which were involved in RTA to find out the boundary of these regions. The positions of breakpoints were between the boundary of the two syntenic regions.

\section{Supplementary information}

Supplementary information accompanies this paper at https://doi.org/10. 1186/s12864-020-07081-0.

Additional file 1: Fig. S1 Circos diagram of the colinearity between $A$. lyrata and C. sativa genomes. Al, A. lyrata; CS, C. sativa. A. lyrata and C. sativa chromosomes are respectively marked in red and green. The syntenic regions which are connected with different Al chromosmes are shown in different colors.

Additional file 2: Table S1 Evaluation of homologous regions between Al and Cs genomes.

\section{Abbreviations}

AK: Ancestral karyotype; ACK: Ancestral crucifer karyotype; PCK: ProtoCalepineae karyotype; tPCK: Translocation Proto-Calepineae Karyotype; BCD: Brassicaceae-common duplications; CNR: Chromosome number reduction; NCF: Nested chromosome fusion; EEJ: End-end joining; RTA: Reciprocal translocation of arms; ADK: Ancestral diploid karyotype of $C$. sativa 


\section{Acknowledgements}

We thank the center for genomics and computational biology lab team for discussion and support.

\section{Authors' contributions}

XW and WW conceived and led the research. ZZ and FM performed the analysis. PS, JY, KG and CL contributed to data analysis and created Python scripts. XW and ZZ wrote the paper. All authors contributed to revising the manuscript. All authors had read and approved the final manuscript.

\section{Funding}

This work was supported by the China National Science Foundation (3117022; Data collection and analysis) to WX, the Ministry of Science and Technology of the People's Republic of China (2016YFD0101001; for writing and publication) to XW.

\section{Availability of data and materials}

The genomes of A. lyrata and C. sativa were downloaded from NCBI (https:// ftp.ncbi.nlm.nih.gov/genomes/all/GCF/000/004/255/GCF_000004255.2_v.1.0, https://ftp.ncbi.nlm.nih.gov/genomes/all/GCF/000/633/955/GCF_000633 955.1_Cs) and the raw data supporting the conclusions of this article will be made available by the authors, without undue reservation, to any qualified researcher.

\section{Ethics approval and consent to participate}

Not applicable.

\section{Consent for publication}

Not applicable.

\section{Competing interests}

The authors declare no conflict of interest.

\section{Author details}

'School of Life Sciences, North China University of Science and Technology, Tangshan 063210, Hebei, China. ${ }^{2}$ College of Pharmacy, Chengdu University of Traditional Chinese Medicine, Chengdu 610075, China. Institute for Genomics and Bio-Big-Data, Chengdu University of Traditional Chinese Medicine, Chengdu 610075, China.

\section{Received: 19 December 2019 Accepted: 18 September 2020}

Published online: 12 October 2020

\section{References}

1. Al-Shehbaz IA, Beilstein MA, Kellogg EA. Systematics and phylogeny of the Brassicaceae (Cruciferae): an overview. Plant Syst Evol. 2006;259(2-4):89-120.

2. Bailey CD, Koch MA, Mayer M, Mummenhoff K, O'Kane SL Jr, Warwick SI, Windham MD, Al-Shehbaz IA. Toward a global phylogeny of the Brassicaceae. Mol Biol Evol. 2006;23(11):2142-60.

3. Koenig D, Weigel D. Beyond the Thale: comparative genomics and genetics of Arabidopsis relatives. Nat Rev Genet. 2015:16(5):285-98.

4. Schranz ME, Lysak MA, Mitchell-Olds T. The ABC's of comparative genomics in the Brassicaceae: building blocks of crucifer genomes. Trends Plant Sci. 2006;11(11):535-42.

5. Mandáková T, Lysak MA. Chromosomal phylogeny and karyotype evolution in $x=7$ crucifer species (Brassicaceae). Plant Cell. 2008;20(10):2559-70.

6. Cheng F, Mandáková T, Wu J, Xie Q, Lysak MA, Wang X. Deciphering the diploid ancestral genome of the Mesohexaploid Brassica rapa. Plant Cell. 2013;25(5):1541-54.

7. Charon C, Bruggeman Q, Thareau V, Henry Y. Gene duplication within the green lineage: the case of TEL genes. J Exp Bot. 2012;63(14):5061-77.

8. Kim C, Wang X, Lee TH, Jakob K, Lee GJ, Paterson AH. Comparative analysis of Miscanthus and Saccharum reveals a shared whole-genome duplication but different evolutionary fates. Plant Cell. 2014;26(6):2420-9.

9. Liu S, Liu Y, Yang X, Tong C, Edwards D, Parkin IAP, Zhao M, Ma J, Yu J, Huang $S$, et al. The Brassica oleracea genome reveals the asymmetrical evolution of polyploid genomes. Nat Commun. 2014;5:3930.

10. Bowers JE, Chapman BA, Rong J, Paterson AH. Unravelling angiosperm genome evolution by phylogenetic analysis of chromosomal duplication events. Nature. 2003;422(6930):433-8.
11. Schubert I, Lysak MA. Interpretation of karyotype evolution should consider chromosome structural constraints. Trends Genet. 2011;27(6):207-16.

12. Wang $X$, Jin D, Wang Z, Guo H, Zhang L, Wang L, Li J, Paterson AH. Telomere-centric genome repatterning determines recurring chromosome number reductions during the evolution of eukaryotes. New Phytol. 2015; 205(1):378-89.

13. Wang Z, Wang J, Pan Y, Lei T, Ge W, Wang L, Zhang L, Li Y, Zhao K, Liu T, et al. Reconstruction of evolutionary trajectories of chromosomes unraveled independent genomic repatterning between Triticeae and Brachypodium. BMC Genomics. 2019;20(1):180.

14. Zhuang W, Chen H, Yang M, Wang J, Pandey MK, Zhang C, Chang WC, Zhang $L$, Zhang $X$, Tang $R$, et al. The genome of cultivated peanut provides insight into legume karyotypes, polyploid evolution and crop domestication. Nat Genet. 2019;51(5):865-76.

15. Moser BR. Biodiesel from alternative oilseed feedstocks: camelina and feld pennycress. Biofuels. 2012;3(2):193-209.

16. Hutcheon C, Ditt RF, Beilstein M, Comai L, Schroeder J, Goldstein E, Shewmaker CK, Nguyen T, De Rocher J, Kiser J. Polyploid genome of Camelina sativa revealed by isolation of fatty acid synthesis genes. BMC Plant Biol. 2010;10:233.

17. Kagale S, Koh C, Nixon J, Bollina V, Clarke WE, Tuteja R, Spillane C, Robinson SJ, Links MG, Clarke C, et al. The emerging biofuel crop Camelina sativa retains a highly undifferentiated hexaploid genome structure. Nat Commun. 2014;5:3706.

18. Martin SL, Smith TW, James T, Shalabi F, Sauder CA. An update to the Canadian range, abundance, and ploidy of Camelina spp. (Brassicaceae) east of the Rocky Mountains. Botany-botanique. 2017;95(4):1-13.

19. Brock JR, Mandakova T, Lysak MA, Al-Shehbaz IA. Camelinaneglecta (Brassicaceae, Camelineae), a new diploid species from Europe. PhytoKeys. 2019;115:51-7.

20. Galasso I, Manca A, Braglia L, Ponzoni E, Breviario D. Genomic fingerprinting of Camelina species using cTBP as molecular marker. Am J Plant Sci. 2015; 6(8):1184-200

21. Chaudhary R, Koh CS, Kagale S, Tang L, Wu SW, Lv Z, Mason AS, Sharpe AG, Diederichsen A, Parkin IAP. Assessing Diversity in the Camelina Genus Provides Insights into the Genome Structure of Camelina sativa. G3: GENES, GENOMES, GENETICS. 2020;10(4):1297-308. https://doi.org/10.1534/g3.119. 400957.

22. Wang J, Sun P, Li Y, Liu Y, Yang N, Yu J, Ma X, Sun S, Xia R, Liu X, et al. An overlooked Paleotetraploidization in Cucurbitaceae. Mol Biol Evol. 2018; 35(1):16-26.

23. Hu TT, Pattyn P, Bakker EG, Cao J, Cheng JF, Clark RM, Fahlgren N, Fawcett JA, Grimwood J, Gundlach H, et al. The Arabidopsis lyrata genome sequence and the basis of rapid genome size change. Nat Genet. 2011; 43(5):476-81.

24. Wang $H$, Ooi BC, Tan KL, Ong TH, Zhou L. BLAST++: BLASTing queries in batches. Bioinformatics. 2003;19(17):2323-4.

25. Wang X, Shi X, Li Z, Zhu Q, Kong L, Tang W, Ge S, Luo J. Statistical inference of chromosomal homology based on gene colinearity and applications to Arabidopsis and rice. BMC Bioinformatics. 2006;7:447.

26. Chen C, Chen H, Zhang Y, Thomas H.R, Frank M.H, He Y, Xia R. TBtools: An integrative toolkit developed for interactive analyses of big biological data Mol Plant. 2020;13(8):1194-202. https://doi.org/10.1016/j.molp.2020.06.009.

\section{Publisher's Note}

Springer Nature remains neutral with regard to jurisdictional claims in published maps and institutional affiliations.

Ready to submit your research? Choose BMC and benefit from:

- fast, convenient online submission

- thorough peer review by experienced researchers in your field

- rapid publication on acceptance

- support for research data, including large and complex data types

- gold Open Access which fosters wider collaboration and increased citations

- maximum visibility for your research: over $100 \mathrm{M}$ website views per year

At $\mathrm{BMC}$, research is always in progress.

Learn more biomedcentral.com/submissions 